\title{
Oscar Niemeyer: Diálogos con lo natural ${ }^{1}$ \\ Oscar Niemeyer: Dialogues with the natural
}

\author{
DOI: 10.17981/mod.arq.cuc.22.1.2019.04
}

Artículo de investigación. Fecha de Recepción: 03/15/2019. Fecha de Aceptación: 04/30/2019.

\author{
Luca Bullaro \\ Universidad Nacional, sede Medellín (Colombia) \\ lucabullaro@hotmail.com
}

Para citar este artículo:

Bullaro. L. (2019). Oscar Niemeyer: diálogos con lo natural, MODULO ARQUITECTURA CUC, vol. 22, no. 1, pp. 83-98, 2019. DOI: http://doi.org/10.17981/mod.arq. cuc.22.1.2019.04

\section{Resumen}

La arquitectura moderna de Brasil ha ido incorporando a partir de finales de los años treinta varios conceptos del Movimiento Moderno Europeo, pero, sin hacerlo de manera directa sino reinterpretando, traduciendo y adaptándose a las especificas condiciones climáticas y paisajísticas del país. Este texto describe la apropiación de las nuevas teorías a partir del análisis de algunos proyectos modélicos en los cuales aparece contundente la concatenación entre espacialidad, paisaje natural y paisajismo. Las obras, realizadas por equipos multidisciplinarios guiados por el maestro Niemeyer, demostrando como la nueva espacialidad brasileña surge de la fusión entre elementos arquitectónicos, artísticos, naturales y estructurales, con el uso de reglas morfológicas de tipo similar en los diferentes aspectos de los proyectos, como sucede por ejemplo en la iglesia Pampulha, donde arquitectos, artistas, ingenieros y paisajistas -Oscar Niemeyer, Candido Portinari, Joaquim Cardoso y Roberto Burle Marx- operan en comunión, aplicando criterios estéticos de naturaleza similar. La revolución brasileña es la asimilación de un método de trabajo fundamentado en el dialogo entre maestros de diferentes saberes, en el cual el arquitecto tiene el importante papel de "orquestador" y como objetivo la construcción holística de un nuevo paisaje moderno, tropical e integrado.

Palabras clave: Niemeyer; Burle Marx; modernidad tropical; arquitectura y paisaje; arquitectura brasileña

\begin{abstract}
The modern architecture of Brazil has been incorporating from the end of the thirties several concepts of the European Modern Movement, but, without doing it in a direct way but reinterpreting, translating and adapting to the specific climatic and landscape conditions of the country. This text describes the appropriation of the new theories from the analysis of some model projects in which the concatenation between spatiality, natural landscape and landscaping appears forceful. The works, made by multidisciplinary teams guided by master Niemeyer, demonstrate how the new Brazilian spatiality arises from the fusion between architectural, artistic, natural and structural elements, with the use of similar morphological rules in different aspects of the projects, as happens for example in the Pampulha church, where architects, artists, engineers and landscapers -Oscar Niemeyer, Candido Portinari, Joaquim Cardoso and Roberto Burle Marx- operate in communion, applying aesthetic criteria of a similar nature. The Brazilian revolution is the assimilation of a working method based on the dialogue between masters of different knowledges, in which the architect has the important role of "orchestrator" and as an objective the holistic construction of a new modern, tropical and integrated landscape.
\end{abstract}

Keywords: Niemeyer, Burle Marx; tropical modernity; architecture and landscape; brazilian architecture

` El articulo es resultado de la Investigación "Poesía y Técnica"del Grupo de investigación: Transepto. 


\section{INTRODUCCIÓN}

El articulo describe algunas diferentes formas de concatenacion entre espacialidad y paisaismo en una serie de obras modélica desarrolladas por Oscar Niemeyer a lo largo de su extensa carrera

La Casa das Canoas y Cavanelas, la iglesia de San Francisco de Asís, el Museo de Arte Contemporáneo (MAC) de Niterói y el auditorio de Ravello son obras, entre muchas otras, que demuestra como la espacialidad moderna de Brasil surge de fusión entre elementos arquitectónicos, naturales y fusión entructurales, con el uso de reglas morfológicas de tipo similar en los diferentes aspectos del proyecto, como acontece por ejemplo en los edificios de Pampulha en los cuales arquitectos, artistas ingenieros y paisajistas -Oscar Niemeyer, Candido Portinari, Joaquim Cardoso y Roberto Burle Marx-operan en comunion aplicando criterios estéticos de naturaleza similar.

Segun Bruno Zevi (1997) el protagonista de la modernidad brasileña es Roberto Burle Marx artista del paisaje, botánico, escritor y cantante. Kenneth Frampton pone en evidencia en varios escritos la extraordinaria creatividad del joven Oscar Niemeyer (Frampton 2009). Pero la importancia de esta revolución moderna no reside tanto en las capacidades de unos pocos si no de la asimilación de un método de trabajo que se fundamentó sobre el dialogo entre maestros de diferentes saberes.

Burle Marx, artista, botánico y paisajista, fue de los primeros que propuso la creación de espacios recreativos y artísticos modernos, realizados a través de elementos vivos, naturales, de diferentes formas, colores y texturas: elementos típicos que bien se adaptaban al clima tropical (Siqueira, 2001).
Niemeyer, influenciado por las ideas y las morfologias amadas del amigo botánico, empezo a partir del final de los años treinta a desarrollar una arquitectura más libre, sensual, natural, orgánica que se concretizó por ejemplo en los proyectos para la Iglesia de Sao Francisco, el Pabellón de New York, la Casa do Baile y la Casa das Canoas, realizados con la colaboración paisajistica de Burle Marx (Bullaro, 2016).

Como afirma Iñaki Abalos (2009), uno de los caracteres distintivos de la arquitectura brasileña deriva de la concatenación de elementos arquitectónicos, naturales y estructurales, y del utilizo de reglas cos, naturales y estructurales, y del utilizo de reglas del proyecto.

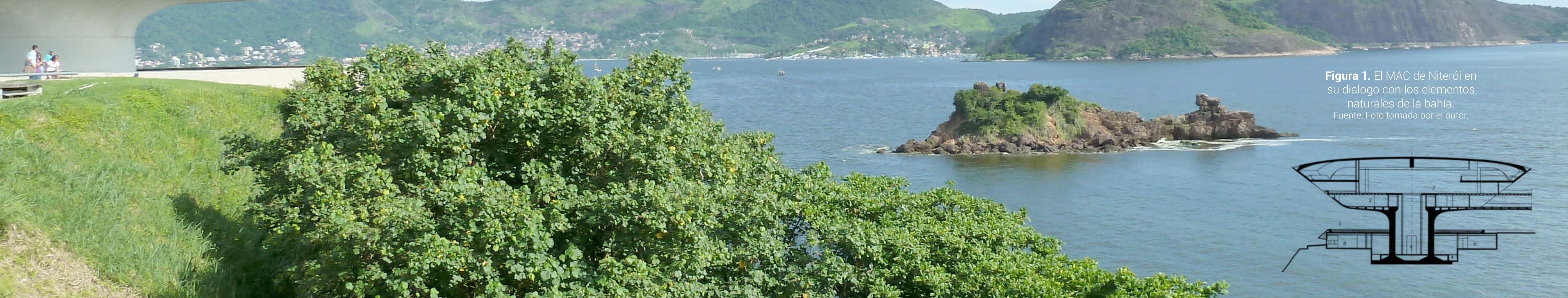


Entre la vasta obra de Niemeyer hay varios proyectos en los cuales se definen reglas morfológicas especificas partir del análisis de los cánones geométricos de los entornos naturales: artificial y organico se relacionan de forma contundente pero do al mismo tiempo.

Con estas reflexiones se quiere poner en resalto algunas características de la metodología de trabajo del maestro a partir del análisis de algunas obras paradigmáticas dos residencia los años cincuenta y dos obras públicas del final de siglo, que representan bien la estrategia proyectua que busca la génesis de una relación extremadamente cercana entre naturaleza y artificio.

\section{Desarrollo}

Niemeyer, a lo largo de su prolífica carrera, ha venido perfeccionando estrategias para conseguir que la arquitectura entablefuertes relaciones con el paisaje natural. El museo de Niteroi, por ejemplo, dialoga de forma poderosa con el entorno: con la poética bahía perfiles sensuates que la circundan.
El texto, a partir del análisis de algunos proyecconjuntos paisajisticos de gran interes, intenta una aproximación a la clasificacion según especificas maneras de interacción con el paisaje.

1. Formas puras, a veces de gran tamaño, que dialogan por contraste con los monumentos geoArmonía formal arquitectura-paisaje a través reinterpreta características formales del entorno (Segre, 2015). gráficos vecinos.

Relación paisajistica generada gracias a la creación de un espacio cuatridimensional minimalista en el cual las rampas externas tienen un pape fundamental en la contemplación del paisaje.

Las obras incluidas en la primera categoría se definen según un juego de repeticiones seriales a partir de la planta o de la sección que generan un enérgico contraste con el entorno, las última dos con la aplicación de geometría curva en planta, en sección, o en las dos al mismo tiempo, que en algunos casos se relaciona, reinterpreta o dialoga de forma contundente, pero respetuosa, con la naturaleza circunstante.

Figura 2. La naturaleza enmarcada en

planta baja del MAC de Niteroi.
Fuente: Foto tomada por el autor.
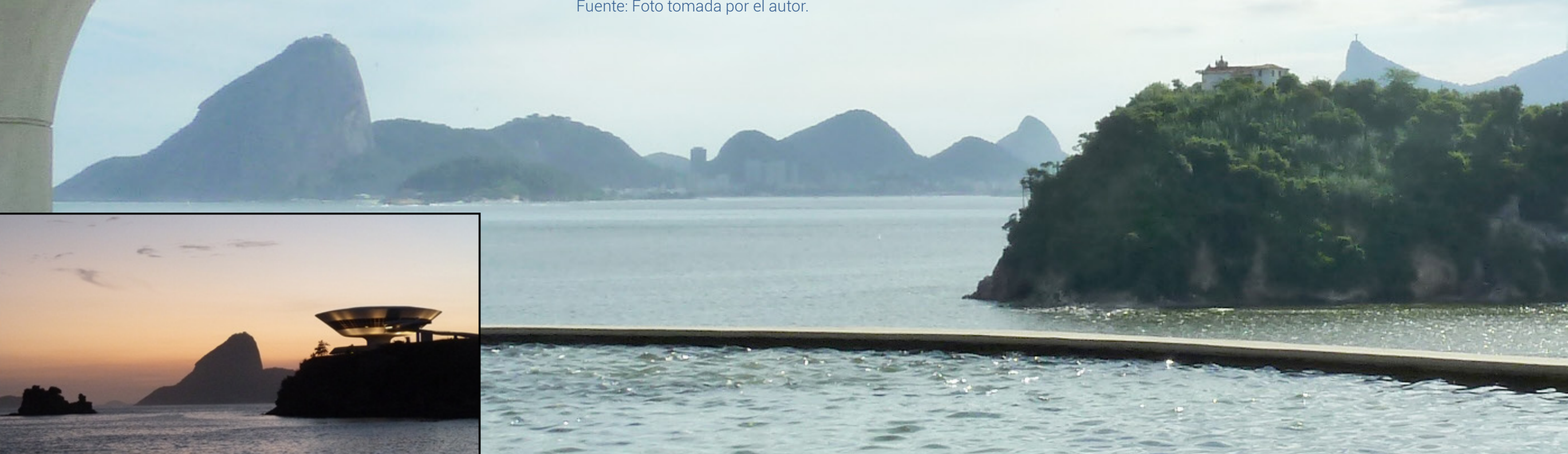

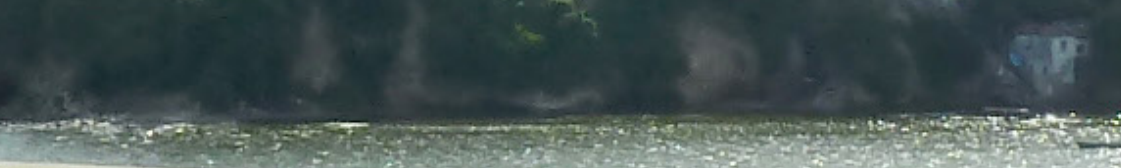
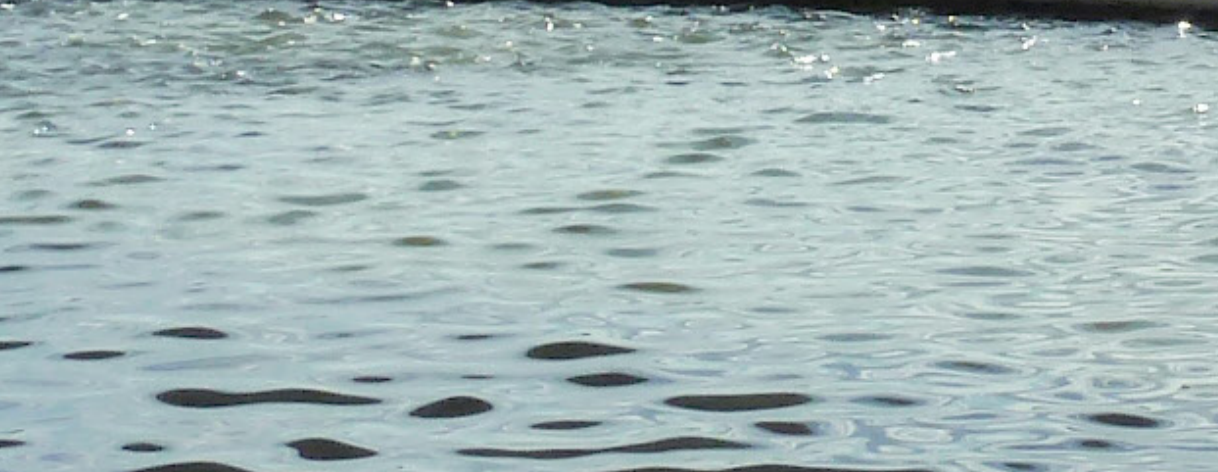

nes

- 
Formas puras y contrastes dialógicos

Este primer tipo se caracteriza por una considerable sencillez: el proyecto no realizado para el Hotel de Petropolis, de 1950, bien representar esta aproximación conceptual: es un volumen puro cuya planta se desarrolla siguiendo un eje principal que presenta una ligera curvatura.

El "basamento abierto" de la planta baja es un mundo complejo de espacios ameboideos que acogun varios tipos de servicios semi-públicos abierto a todos los visitantes (Papadaki, 1956). El volumen dimensiones monumentales, crea un fuerte contraste con las montañas cercana, crea un fuerte contraste malismo, proporciona una sensación de respetuos discordancia que recuerda aquella trasmitida por el volumen puro de la roja casa Malaparte -Adalberto Libera- encajada en la espléndida península de roca gris de la isla de Capri (Saravia, 2010).

El Hospital de Rio de Janeiro, a pocos metros del jardín botánico, sigue el mismo esquema, peroa dertir de una geometría de rectángulos puros. Parece que el método proyectual del maestro está enfocado a no quitar protagonismo simbólico y estético al vecino quilar protagonis sósimportantes monumentos naturales de la ciudad Se entabla de nuevo un respetuoso contraste con lavecina montaña y también el cuerpo secundario cubierto por una enorme bóveda blonca que se podrí interpretar como una reinterpretación de los perfiles del cerro: una poderosa sección tación de los pefles del cerro una podesa sección en el suelo del jardín de Burle Marx (Papadaki, 1956).

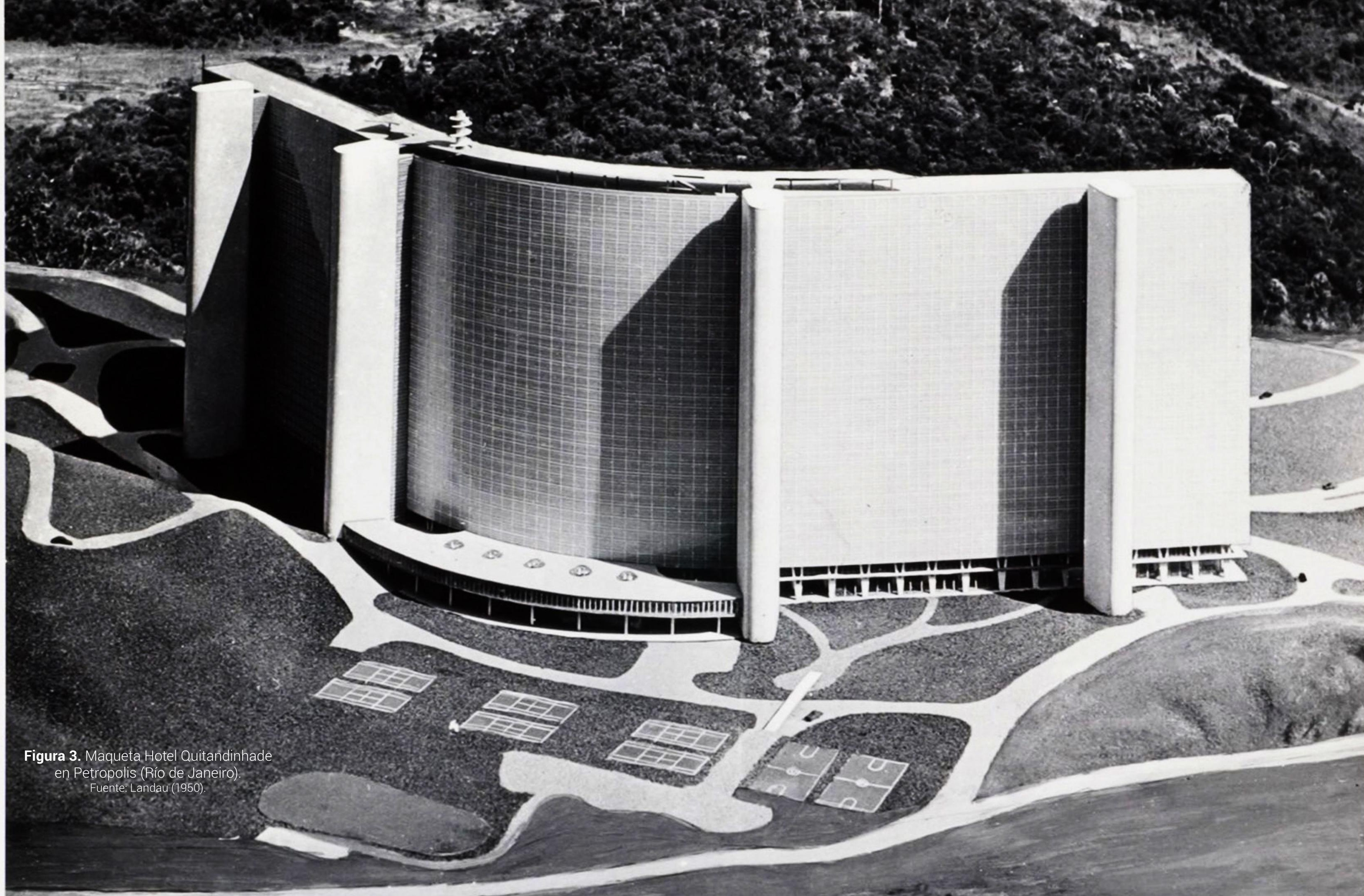




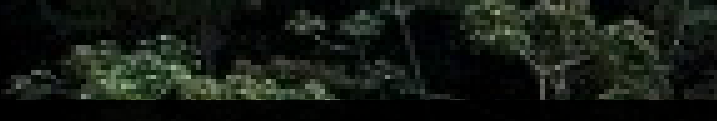

Tipo dos

Armonía formal con el entorno:

Biomorfismo

En la casa Cavanelas la colaboración entre Niemeyer y Burle y Burle Marx genera una obra que se inserta en $e$

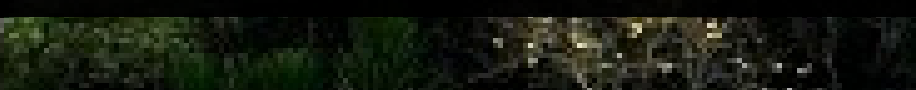

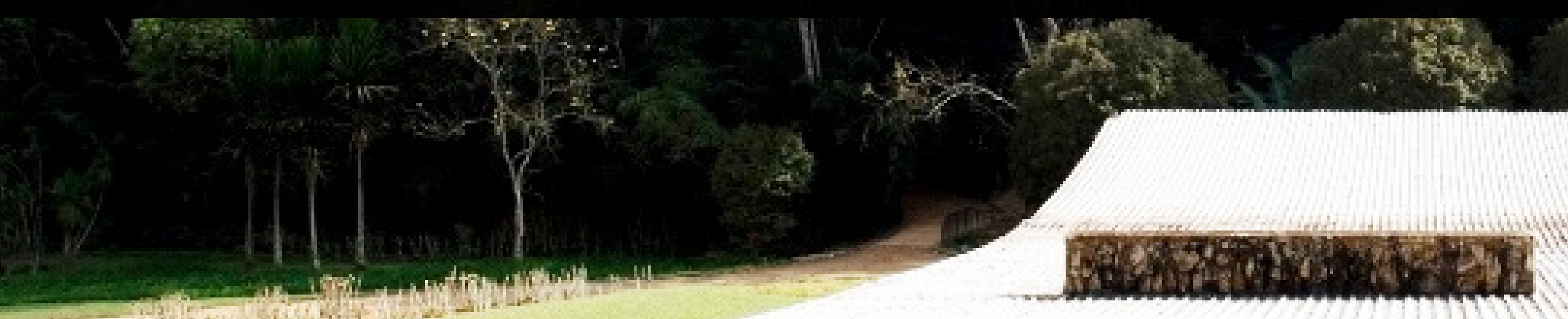

al mismo tiempo: una bisagra artificial en forma de catenaria que conecta visualmente las dos laderas del valle y permite apreciar desde un punto de vista privilegiado la perfección de los dos jardines uno "geométrico" y el otro "orgánico" (Bullaro. 2017)

a)
La famosa cubierta de la Casa das Canoas -que protege los espacios interiores de las lluvias tropicales y de los intensos rayos del sol- configura un abrazo arquitectónico en forma de ameboide que acoge los usuarios y la grande piedra: la naturaleza se engloba a la arquitectura. El monolito central es una bisagra espacial de conexión entre cubierta entre interior y exterior, en el cual los elementos naturales y artisticos en forma de esculturas, definen una espacialidad que reinterpreta las reglas biológicas y geológicas del extraordinario entorno dando vida a una obra holística y emblemática que como escribe Alvar Aalto "es una flor exquisita que crece en un medio encantado" (Aalto, 2000)
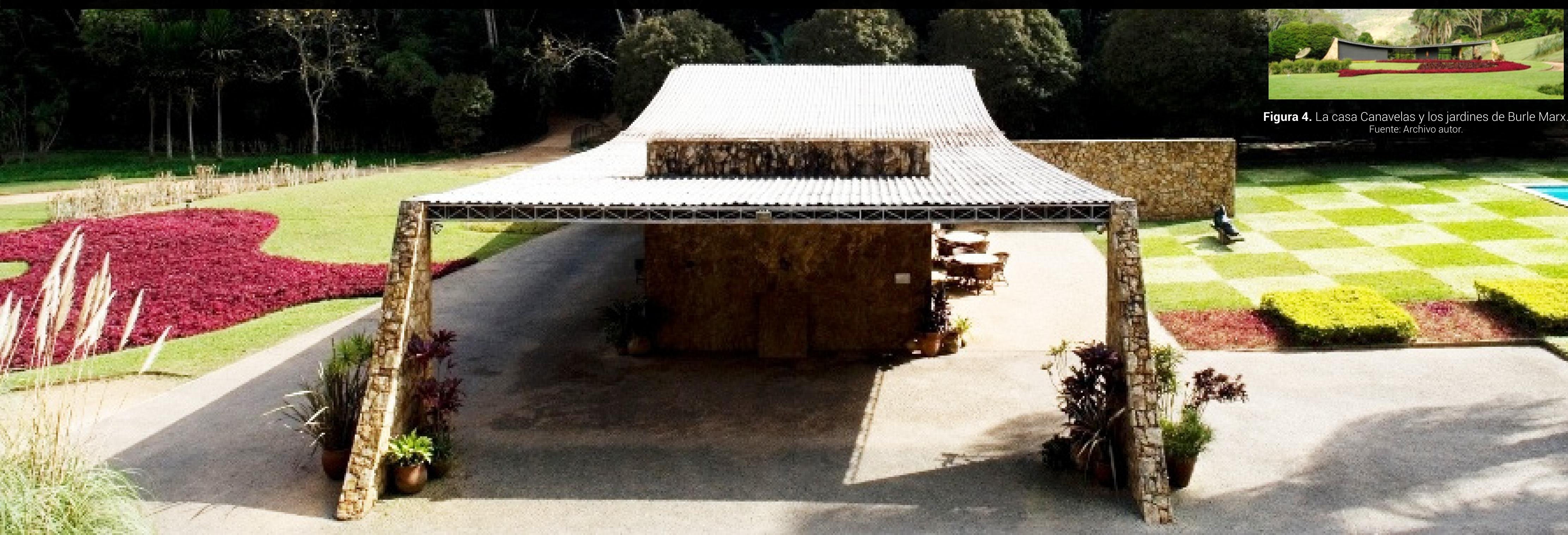

Figura 4. La casa Canavelas y los jardines de Burle Marx

. 
Espacios cuatridimensionales minimalistas

El Museo de Arte Contemporáneo de Niteroi y el Auditorio de Ravello, realizados más recientemente, son dos ejemplos contundentes de relación holística entre elementos naturales y artificiales. El museo se encuentra a pocos kilómetros de Rio de Janeiambiente geomorfológico de gran impacto estético y emocional.
El Museo de Niteroi, al cual la crítica internacional considera una de las obras arquitectónica más importante de los últimos años, brota de una penínsude roca grises (Maciel, 2007). Sobre su solemne tronco-columna se levanta y se curva para definirlas "ramas" que se despliegan en forma de una extensa bandeja blanca en voladizo. El intradós que cubre y protege el espacio de la planta baja se pliega a formar la fachada principal, a tana corrida única. No hay fachas si no fachada, no hay columnas si no co- mo ayuda en la génesis de una relación con el lugar decidida pero respetuosa. El paisaje se enmarca en parte en la planta baja y se desdobla y refleja gracias al espejo de agua y a través de la extensa ventana continua.

La rampa tiene un poderoso papel didáctico en la forma de relacionarse con el entorno: es un dispositivo que nos obliga a multiplicar nuestro punto de vista, que va constantemente rotando y cambiando de inclinación y de altura hasta llegar al acceso principal del museo (Niemeyer, 2004).

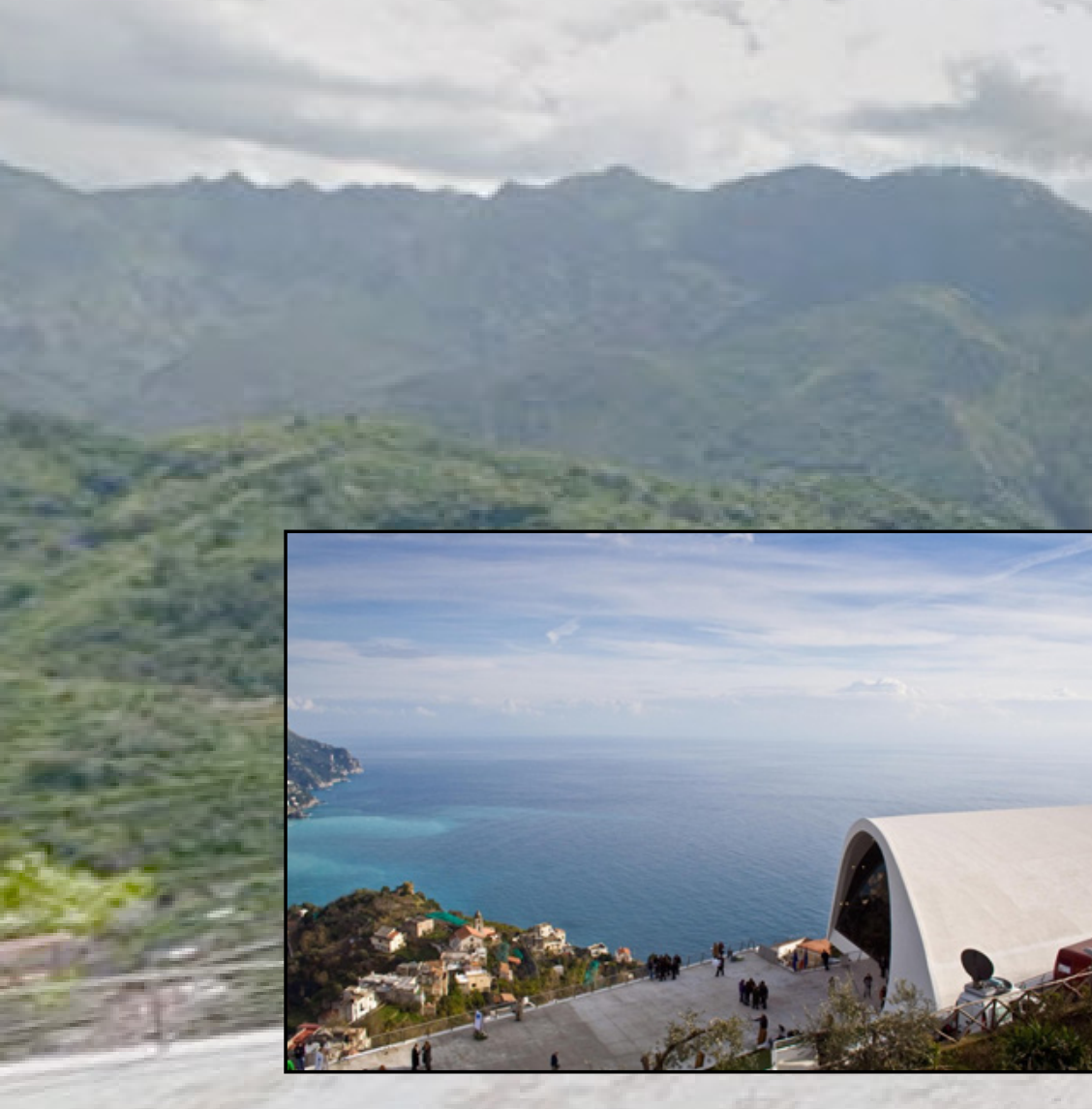

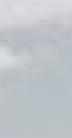


Penetrando en el espacio interior, oscuro, la relación con el entorno cambia por completo. Estamos en un lugar protegido. Los vidrios apuntan hacia abajo, a las delicadas olas de la bahía Gracias a la inclinación de la pared y de los vidrios, los rayos del sol que se reflejan en el espejo de se proyectan en el intradós de la cubierta. La inclinación tiene la función de proteger de los rayos directos del sol: en correspondencia al amanecer atardecer cuando logran penetrar generando un uego de sombras curvas de extraordinaria inten-

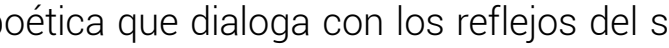
que provienen de la superficie de la bahía.
En el proyecto para el Auditorio de Ravello, de año 2000, como en el MAC de Nieteroi, Niemeyer concibe un elemento arquitectónico enérgico, en respetuoso dialogo con el paisaje. Se utiliza una estrategia clara: apoyar un prisma escultórico

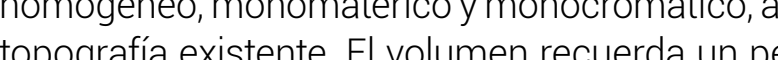

con ojos asimétricos: uno redondo, de pequeñas dimensiones el otro de forma lenticulary de mayo tamaño, que proporciona el acceso al auditorio y que permite una visión enfática de la línea de la horizonte. La bóveda cóncava genera Figura 6. Auditorio de Ravello (Italia).
Fuente: 0 Google Earth.

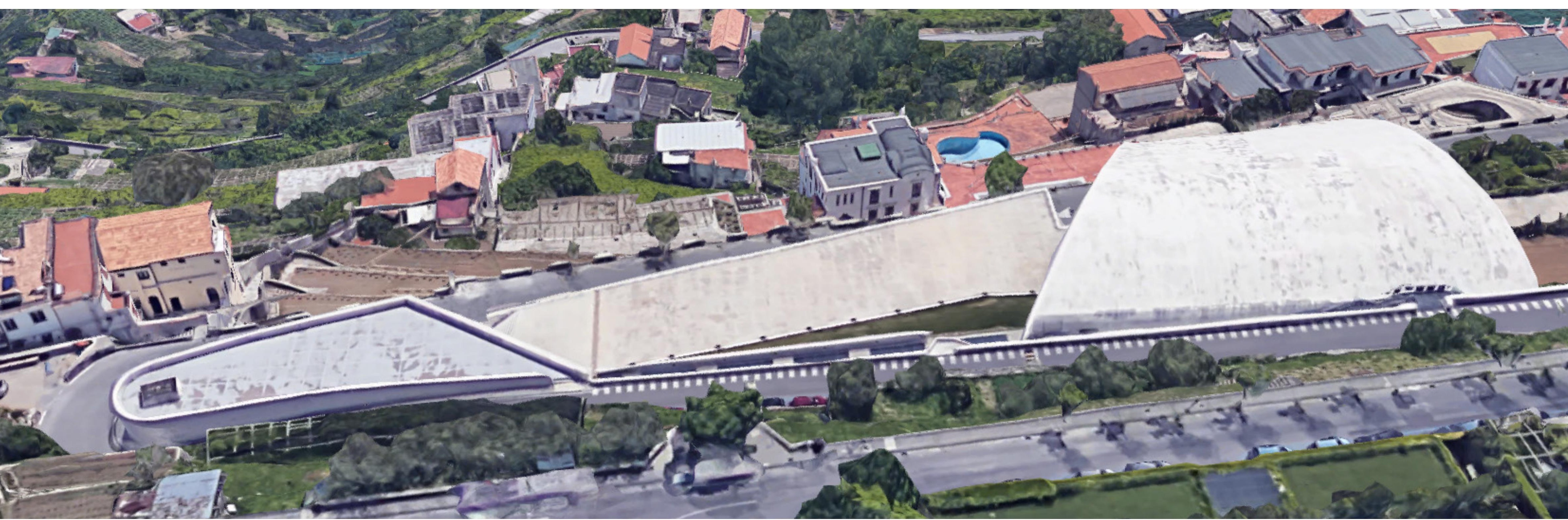



blanco en la ardua pendiente $y$ pouadrar el magnifico panorama, generando un poética dicotomía entre la clara bóveda y el sistema de rocas grises que configuran la segmentada linea El maestro escribe: "dentre os projetos que elaborei nesses dez anos, o Teatro de Ravello foi um dos que mais me interessaram, por se tratar de um projeto para o exterior numa das cidades mais lindas da Itália, num terreno debruçado sobre o mar." (Niemeyer, 2014)
El blanco del revoque y la forma del techo rememoran los conjuntos habitacionales cubiertos con bovedas típicos de algunas islas del Mediterráneo, en los cuales las superficies blancas parecen acariciar el cielo y dialogar con la masa de las nubes. La uz mediterránea, idolatrada por Le Corbusier, Aalto Gaudí, contribuye de manera contundente a ani. ficies encaladas con los claroscuros de sus sombras.

El volumen escultural se apoya en una pequeña pendiente de la colina: una estrategia conceptual con algunos puntos en común con la ensayada en a Casa das Canoas y en el MAC de Niteroi (Bullaro, 2014). Existe también una similitud conceptual con las terrazas de la Casa Kaufmann de Wright que proyectan en voladizo hacia la naturaleza.

Estos dos proyectos, el museo y el auditorio, 作 definición previa de la sección, son instrumentos de
Niteroi la contundencia aparece poderosa: es una relación total, en trescientos sesenta grados; en el caso del proyecto italiano hay un doble dialogo, con el paisaje lejano, del horizonte marino, y una relación más cercana y más íntima con la pendiente de la ladera, con sus antiguas construcciones y su típica vegetación

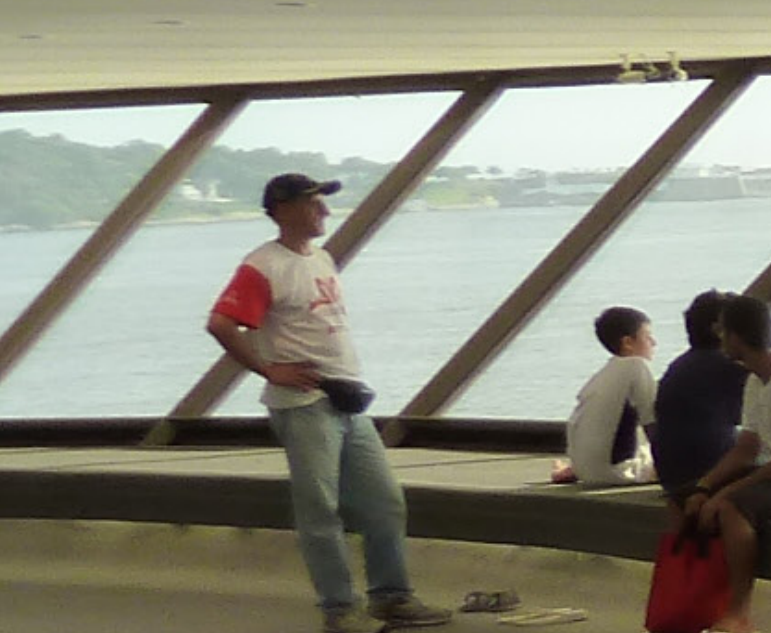

Figura 7. La ver I MAC de Niteroi del MAC de Niteroi.
Fuente: Foto tomada por el autor.
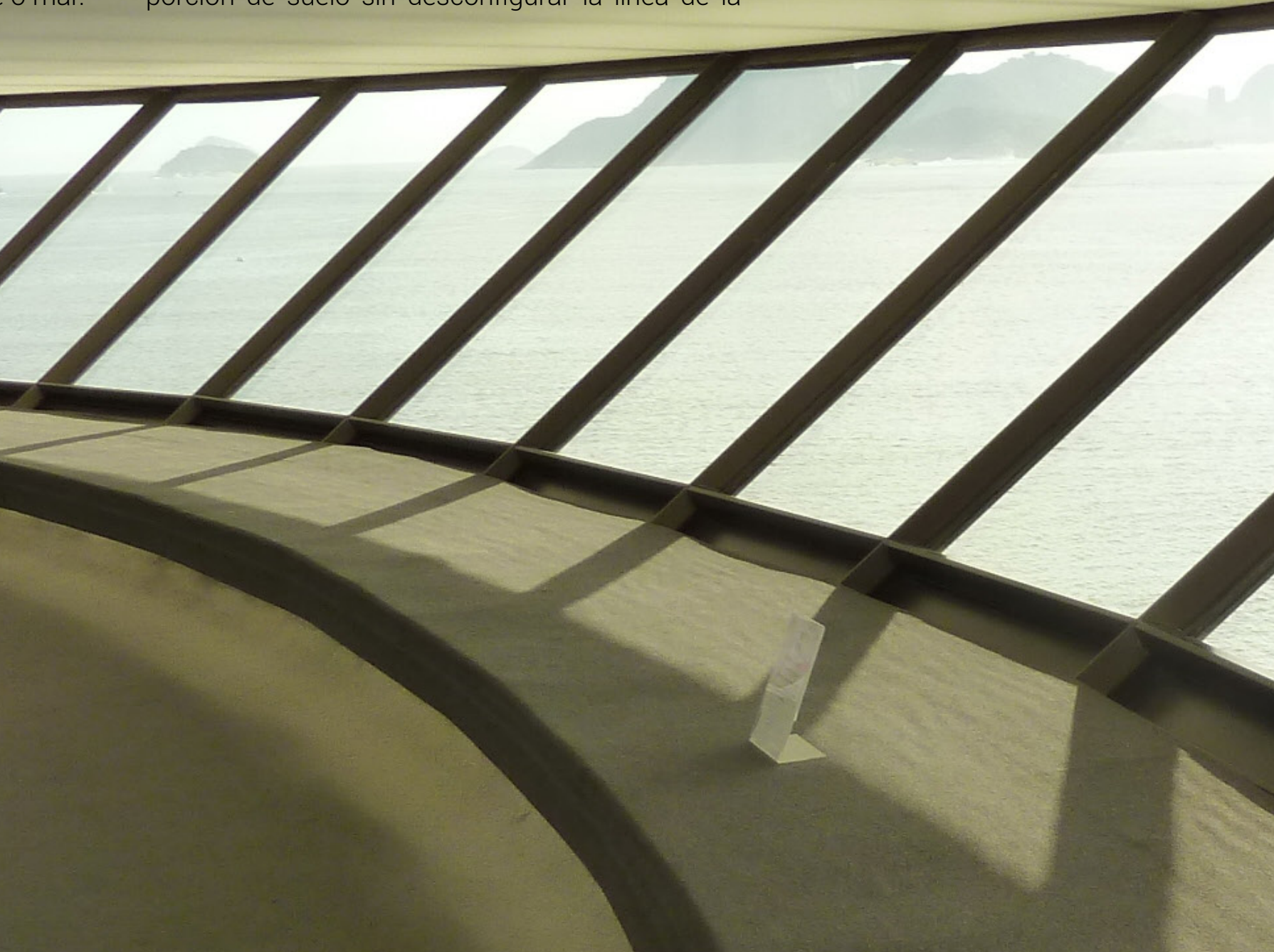
En estas dos obras se abandona casi por completo la predominancia de líneas rectas y de ángulos de noventa grados. Se define un sistema de cuerpos tridimensionales orgánicos, blancos, que a veces se desdoblan en espejos de agua, acompañados en la mayoría de los casos por unos "tentáculos espaciales" que permiten, como en las antiguas villas barrocas, la conexión entre la cota cero e "il piano nobile", el piso superior, más panorámico. Caminos cuatridimensionales que facilitan al mismo tiempo una experiencia plurisensorial en la cual domina el tema de la apreciación y del descubrimiento del paisaje -de los elementos naturales y de la misma arquitecturadesde dinámicos y multíplices puntos de vistas.

\section{Conclusiones}

Como manera abierta de finalizar estas reflexiones se quiere subrayar las enseñanzas múltiples, y muy actuales, y como la metodología de trabajo empleada en estas obras brasileñas es trasmitida. Enseñanzas que poseen una importante congruencia y actualidad. Podemos resumir algunos importantes conceptos derivados de este análisis.

La correspondencia en las reglas morfológicas de la arquitectura con el entorno: varios principios formales que se hallan en los perfiles de las montañas, las colinas y las orillas de Brasil se reinterpretan en los trazos de la arquitectura y los jardines adyacentes.

El dialogo constructivo: fantasioso y creativo experimentador; Niemeyer actúa como un director de orquestra consintiendo la sapiente fusión entre formas, espacios y paisaje. Las colaboraciones multi- disciplinarias son la base de la estrategia de trabajo y de los excelentes resultados en varios de los más interesantes proyectos del Moderno de Brasil.

El minimalismo: tema típico de la poética de Niemeyer; muy a menudo usado para lograr la integración entre elementos naturales y artificiales. Un proceso de purificación formal que facilita descartar conceptos, colores y materiales innecesarios para generar un intenso dialogo con los poderosos monumentos naturales de Brasil. La sencillez es un objetivo preciso del maestro carioca: genera pureza, favorece la incorporación y facilita la fusión entre espacio y paisaje.

La Modernidad de Brasil se configura entonces como revolucionaria y tropical por la concatenación atenta de diferentes aspectos provenientes del mundo de las artes y del paisaje autóctono. Ha desarrollado, gracias a la reinterpretación de cánones morfológicos del paisaje típico, un amplio conjunto de novedosas grafías que surgieron de la orquestación del trabajo analítico y proyectual a partir de grupos multidisciplinarios en los cuales estaba presente la sabiduría botánica y artística de Burle Marx: generó así una sabia estrategia de intervención que aparece de extrema actualidad, sobre todo en estos últimos años de perversas transformaciones de los entornos naturales en todo el planeta.

\section{REFERENCIAS}

Aalto, A. (2000). La Casa de Oscar Niemeyer en las afueras de Río de]aneiro. En, G. Schildt, A. Góran. Alvar Aalto. De palabra y por escrito. El escorial: El croquis. 
Abalos, I. (2009). Os rabiscos de Niemeyer. En, R. Segre, Tributo a Niemeyer, (56-61). Rio de Janeiro: Viana.

Bullaro, L. (2017). Dos casas tropicales. Iconofacto, 13(20). 229-250.

Bullaro, L. (2016). Brasil Holístico. Seminario XI, DOCOMOMO internacional. Brasil, Recife.

Bullaro, L. (2014). Moderno y tropical: la reinterpretación de los principios lecorbuserianos en las primeras obras de Oscar Niemeyer. DeArq, 15(1). 36-51.

Frampton, K. (2009). Homenagem a Niemeyer. En, R. Segre, Tributo a Niemeyer. (26-27). Rio de Janeiro: Viana.

Maciel, F. (2007). La vida es un soplo, Gavea Filmes, Wavelenght, Brasil.

Niemeyer, O. (2004). Minha arquitetura. Río de Janeiro, Revan.

Niemeyer, O. (2014). Auditorio de Ravello Memoria del proyecto. En la página web de la fundación Niemeyer: www.niemeyer.org.br. Consultado el 25-09-2017.

Papadaki, S. (1956). Oscar Niemeyer, Works in progress. New York: Reinhold Publishing Corporation.

Saravia, G. (2010). Los dos mundos en Casa Malaparte. Dearq, 7(1).96-111. https://doi. org/10.18389/dearq7.2010.09

Segre, R. (2015). Paradojas estéticas de un Niemeyer definitivo. En, R. Segre, Arquitectura y urbanismo. (347-354). La Habana: arte y literatura.

Siqueira, V. (2001). Burle Marx. São Paulo: Cosac e Naify.

Zevi, B. (1997). Storia e controstoria dell'architettura in Italia. Roma: Newton.
Luca Bullaro es Doctor en Proyectos Arquitectonicos - Ph.D. de la Università degli studi di Palermo, Italia, en cotutela con el doctorado "Proyectos arquitectónicos" Universidad Politécnica de Catalunya (Barcelona). Magister en "Arquitectura: Critica y Proyecto" de la UPC-ETSAB, Barcelona (España). Y profesor asociado de la Universidad Nacional de Colombia, sede Medellin.

A partir de 2002 gana diferentes premios y concursos en Italia y en el extranjero. En 2012 gana el concurso para el proyecto de la "Plaza Fundadores" en el campus de la Universidad Pontificia Bolivariana (UPB) de Medellín (Colombia), desarrolla el proyecto de la Ciudadela habitacional Comfenalco en Apartadó (Colombia). En 2016 gana el concurso para la realización de la iglesia de santa Barbara en Licata (Italia).

Entre 2011 y 2016 desarrolla las investigaciones "Arquitectura contemporánea en Medellín" sobre el desarrollo sostenible e incluyente de la ciudad; "Arquitectura para la democracia" sobre la obra de Josep Lluis Sert y "Poesía y Técnica" (en fase de publicación) sobre la poética de Oscar Niemeyer.

$\mathrm{Ha}$ expuesto sus proyectos en Ferrara (Premio Biagio Rossetti, 2003), en Roma y Barcelona (NIB-ICAR 2004), en Chicago (Sicilia Olanda, 2007), en la Triennale di Milano (Medaglia d'Oro all'Architettura italiana, 2009), en el Design week (Estambul, 2009) y en la VI Bienal Europea de Paisaje (Barcelona, 2010). Ha dictado conferencias en Roma, Alicante, Barcelona, Manizales, Cali, Bucaramanga, Bogotá, Santiago de Chile, Valparaíso, Buenos Aires, Ciudad de México, Rio de Janeiro, Recife y La Habana. 